\title{
Propuesta de un modelo orientado a la intención de ampliar servicios de ahorro y crédito bancarios: una aplicación al mercado de clase baja de Monterrey, Nuevo León
}

\author{
Nydia Soledad Peña Vela / npv_garza@hotmail.com \\ Universidad Regiomontana \\ Judith Cavazos Arroyo / judith.cavazos@upaep.mx \\ Universidad Popular Autónoma del Estado de Puebla \\ Ana Paola Sánchez Lezama / paolas25mx@yahoo.com.mx \\ Universidad Popular Autónoma del Estado de Puebla
}

\section{RESUMEN:}

Esta investigación exploratoria identificó factores que influyen en la intención de ampliar servicios bancarios. El poder predictivo del modelo propuesto es evaluado con PLS (por sus siglas en inglés), en clientes bancarios de clase baja en Monterrey, México. Los resultados muestran que la intención de ampliación de servicios es afectada negativamente por la intención de cambio de banco, y que la percepción de las estrategias vinculadas a los aspectos tangibles de ahorro e intangibles de crédito y la actitud hacia los empleados y los servicios afectan indirectamente la formación de dicha intención. La satisfacción no resulta significativa hacia tal ampliación de servicios.

Palabras clave: ampliación de servicios bancarios, estrategias orientadas a incentivar ahorro y crédito, clase baja

\section{ABSTRACT:}

This exploratory research identified factors which influence the intention to expand bank services. The predictive power of the proposed model is evaluated with PLS among low-income bank clients in Monterrey, Mexico. The results indicate that the intention of expanding services is negatively affected by the intention to switch banks, and that the perception of the strategies related to tangible savings and intangible credit aspects and the attitude toward the employees and services indirectly affect the formation of said intention. Satisfaction regarding expanded services is not significant.

Keywords: expanded bank services, strategies oriented to stimulate savings and credit, low-income class 


\section{INTRODUCCIÓN}

Las causas del ineficiente acceso a los servicios bancarios en varios países de América Latina abarcan una amplia variedad de aspectos económicos y sociales, además de aquellos relacionados únicamente con el sistema financiero. La baja calidad en la atención del cliente, la demora en el tiempo de espera y la insuficiente información sobre los servicios financieros disponibles constituyen ejemplos de ineficiencias en la provisión de servicios bancarios (Rojas, 2006).

Es importante que un sistema financiero formal opere en todos los sectores de la población proporcionando servicios financieros básicos. A partir de la reforma del año 2007, llevada a cabo por la Cámara de Diputados para la creación de "bancos de nicho", en México se reestructuró la oferta bancaria bajo un enfoque de segmentación de mercados basado en niveles de ingreso, ofreciendo servicios limitados bajo el concepto de bancos-tienda (Zamarripa, 2008), y estimulando con ello la bancarización de segmentos que tradicionalmente habían sido excluidos del sistema financiero formal, vía el acceso y el fomento del uso de servicios financieros (Garrido, García y Morales, 2011). Estos bancos, enfocados en el segmento de la clase baja del país, pretenden que los clientes que se han bancarizado adquieran más productos financieros, además de atraer a nuevos consumidores que no han sido atendidos por la banca múltiple tradicional. El objetivo es ofrecer soluciones de financiamiento a su medida para la adquisición de créditos de consumo (autos vivienda, electrodomésticos, muebles, ropa, etc.) y, también, opciones financieras de capital de trabajo o de inversión.

En México, el 65\% de la población representa el mercado potencial de los nuevos jugadores financieros, ya que este mercado ha financiado tradicionalmente sus necesidades de consumo a través de empresas comerciales (Sigmarket, 2008). La tienda comercial financia alrededor del $75 \%$ de personas de bajos ingresos y al $25 \%$ de ingresos más altos. Los bancos, por el contrario, le han prestado mayormente al nivel socioeconómico de ingresos más altos y muy poco al nivel correspondiente de baja renta (Carbajal, 2008). Recientemente, varios de los denominados "nuevos bancos" han estado promocionando productos y servicios orientados al mercado de cla- 
se baja en el país, creciendo muy rápidamente en captación, ahorro y crédito, operando con tasas bajas para los depósitos y altas para los créditos (Garrido y García, 2009). Banco Wal-Mart de México Adelante, por ejemplo, visualiza un mercado potencial en los $2.5 \mathrm{mi}$ llones de clientes que acuden diariamente a las tiendas de autoservicio del grupo, a quienes pretenden ofrecerles productos de ahorro, desde los $\$ 4.5$ dólares, y crédito hasta por $\$ 180$ dólares, pagaderos en 24 meses (Galán, 2007).

Este trabajo, enfocado a clientes bancarizados de clase baja, tiene dos objetivos: primero, identificar y recabar los factores reportados en la literatura que pueden influir en la ampliación de servicios bancarios y segundo, aportar al estudio del comportamiento del consumidor mexicano un modelo que contribuya a una mayor comprensión del proceso de intención de ampliar los servicios bancarios por parte de clientes de bajos ingresos.

\section{REVISIÓN DE LA LITERATURA}

\section{INTENCIÓN DE AMPLIAR SERVICIOS BANCARIOS}

Uno de los factores más importantes para determinar la conducta de una persona es la intención, que se refiere a la disposición a realizar una conducta determinada y funciona como un predictor de ésta. La intención es afectada por la actitud, la situación actual y las probabilidades de ciertas circunstancias (Hellier, Geursen, Carr y Richard, 2003). La probabilidad de ampliar los servicios bancarios de los clientes, se debe a la satisfacción y cierto tiempo de relación con el banco, a pesar del riesgo que implica tener operaciones con una sola institución. Un cliente estaría dispuesto a continuar o incrementar los servicios del banco principalmente por la percepción de la calidad del servicio, así como la duración de la relación. Sin embargo, también otros factores como la percepción del costo de cambio de banco, el apego emocional a la institución bancaria y, en menor medida, las políticas de precio (tasa de interés en depósitos y créditos) inciden en el uso y en la selección del banco (Lam y Burton, 2006). La percepción es definida como el proceso a través del cual el individuo selecciona, organiza e interpreta estímulos para formar una imagen significativa y coherente del mundo (Schiffman y Kanuk, 2001). 
En este estudio, estos factores fueron incluidos desde la perspectiva de la percepción de estrategias bancarias de ahorro (PEBA), la percepción de estrategias bancarias de crédito (PEBC) así como la actitud hacia los servicios del banco (ASB), la satisfacción percibida por los clientes (SPCB) y la intención del comportamiento de cambio de banco [Bank Customer Switching Behavior Intention] (ICCB).

\section{ESTRATEGIAS BANCARIAS DE AHORRO Y CRÉDITO}

Los proveedores de servicios financieros necesitan usar estrategias claras tanto para la elección como la retención de clientes apoyándose de sus atributos clave. Esto requiere el desarrollo de productos específicos, programas de ventas cruzadas (Lam, Burton y Lo, 2009), uso de tecnología (Mercado, 2007), variedad y diferenciación de productos (Lovelock, 1997; Hoffman y Bateson, 2006). Smith (2000) sugiere también considerar aspectos de acceso y conveniencia, factor humano y tangibles. Dado que la percepción del cliente sobre las características del producto o servicio se relaciona directamente con la actitud a la compra de dicho bien (Gammacurta, 2006), es recomendable desarrollar estrategias de servicio bancario de ahorro y crédito focalizadas en diferenciar los atributos tangibles e intangibles vinculados a estos productos.

\section{ACtitud haCIA LOS SERVICIOS DEL BANCO}

La actitud se refiere a la disposición que muestran las personas hacia ciertas situaciones, la cual influye en la realización de las actividades y permite facilitar u obstaculizar los procesos en el servicio (Valencia, 2005). Tanto la actitud como la satisfacción anteceden la intención (Bearden, Netemeyer y Teel, 1989), pero el nivel de satisfacción bancario es el resultado de las percepciones del cliente, las actitudes desarrolladas y el nivel de uso asociado, tanto con la banca automatizada como de persona a persona (Murdock y Franz, 1983, Moutinho y Meidan, 1989; Moutinho, 1992; Moutinho y Smith, 2000).

\section{SATISFACCIÓN PERCIBIDA POR LOS CLIENTES}

La satisfacción se define como un estado emocional que resulta de la interacción del cliente con un proveedor de servicios a través del 
tiempo (Crosby et al., 1990). Algunos autores (Bitner y Hubbert, 1994; Johnson y Gustafsson, 2000; Oliver, 1997) se han enfocado en investigar la relación entre calidad y satisfacción, encontrándose que ambas están estrechamente relacionadas (Bitner y Hubbert, 1994; Dabholkar, 2000). Los efectos de las actitudes de los clientes bancarios hacia la intención de ampliar los servicios, la lealtad y el cambio de banco están mediados por la satisfacción percibida (Moutinho y Smith, 2000). Se espera que las actitudes impacten sobre la satisfacción y esta afecte la lealtad hacia el banco y, por lo tanto, también la intención de cambio de banco. De hecho, la satisfacción es considerada el aspecto más relevante en este tipo de relaciones, ya que determina tanto el comportamiento de cambio de banco por parte del cliente como los patrones de lealtad y retención (Moutinho y Meidan, 1989; Manrai y Manrai, 1993).

\section{INTENCIÓN DE CAMBIO DE COMPORTAMIENTO}

Pueden ser varios los aspectos que inciden sobre la insatisfacción de un cliente y lo lleven a considerar cambiar de comportamiento; en este caso, reflejado por la intención de cambiar de banco. La gerencia, si desea influir en el cambio de comportamiento y evitar la deserción, debe entender la conducta de sus clientes, mejorando así las relaciones de largo plazo con estos (Clemes, Gan y Zheng, 2007). Por parte del cliente, la intención del cambio de comportamiento significa abandonar a un proveedor de servicios por otro (Garland, 2002). Aspectos como el precio, los inconvenientes, las fallas en los encuentros y respuesta de servicio, la ética, la competencia y los cambios involuntarios incrementan la posibilidad de cambio de comportamiento (Keaveney, 1995). Aunque puede asumirse que un incidente crítico influye en la intención de cambio de proveedor del servicio, algunos investigadores consideran que la deserción también puede ocurrir cuando el consumidor enfrenta varios problemas en el tiempo de relación con el proveedor (Bejou y Palmer, 1998; Hocutt, 1998). 


\section{Método}

\section{Modelo teÓRICO}

A continuación se propone el modelo teórico con las hipótesis correspondientes, realizado a través de la investigación basada en la literatura, respecto a las variables relacionadas con la intención de ampliar servicios con el banco (Figura 1).

Figura 1

Modelo conceptual de ampliación a la bancarización

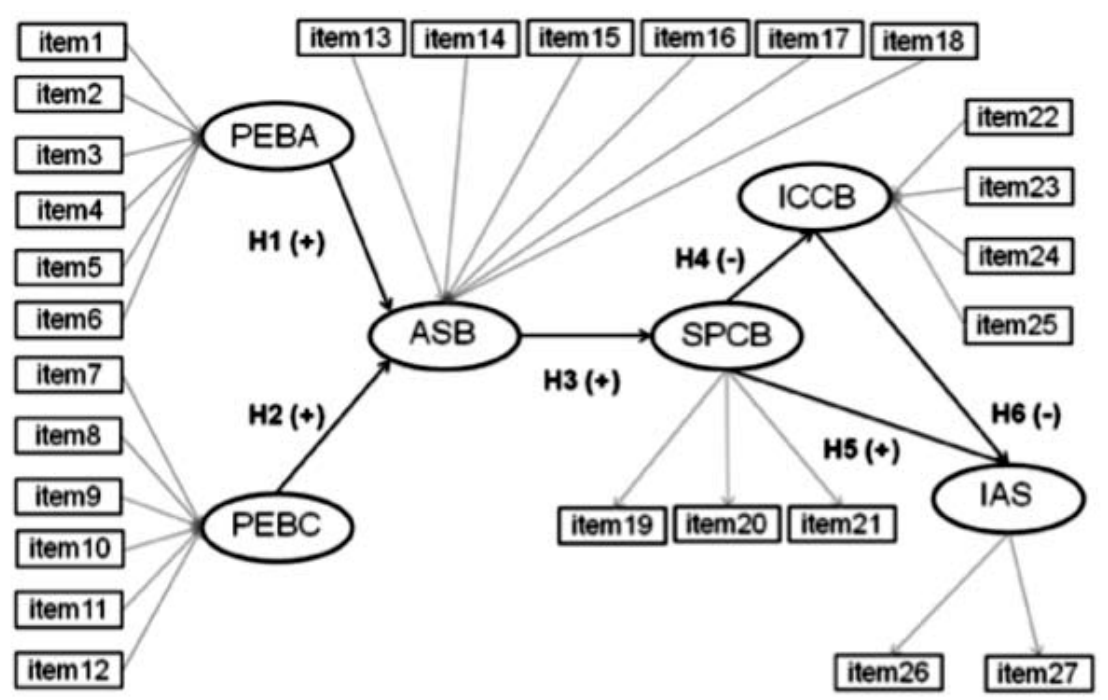

PEBA $=$ Percepción de estrategias bancarias de ahorro hacia el banco. $\mathrm{PEBC}=$ Percepción de estrategias bancarias de crédito hacia el banco. $A S B=$ Actitud hacia los servicios de banco. SPCB = Satisfacción percibida por los clientes del banco. ICCB =Intención del comportamiento de cambio de banco por parte del cliente. IAS = Intención para ampliar servicios.

\section{Diseño y Metodología}

En este estudio, los datos empíricos fueron obtenidos a través de un método de selección no probabilístico por conveniencia, con una muestra de 200 personas bancarizadas, que viven en la ciudad de Monterrey capital del estado de Nuevo León, en México. Dichas personas fueron interrogadas respecto a su intención de ampliar los servicios con su banco y respecto a la evaluación de diversos factores 
identificados en la revisión de la literatura, utilizando escalas Likert de 5 puntos (donde el valor 5 representa la respuesta más positiva). Tales escalas fueron previamente seleccionadas, pre-examinadas con una prueba piloto, y adaptadas al contexto (Apéndice I - Tabla 1).

La definición de la naturaleza de los constructos fue verificada por medio de través de la lista de criterios y características propuesta por Fassott (2006) y Jarvis, Mackenzie y Podsakoff (2003); cuatro constructos fueron modelados como formativos (PEBA, PEBC, ASB, ICCB) y el resto como reflectivos.

Dado que procedimientos para escalas de intervalo pueden ser aplicados a la escala Likert con 5 a 7 categorías (Garson, 2009a), la influencia de los casos con un valor extremo univariado (aquellos puntajes mayores a 3 desviaciones estándar a partir de la media) fue reducida, convirtiéndolos a valores equivalentes al puntaje extremo más cercano que se encuentre dentro de 3 desviaciones estándar de la media (Kline, 2010).

Para evaluar el poder predictivo del modelo teórico, modelos de ecuaciones estructurales (MEC o SEM por sus siglas en inglés), en específico la técnica de mínimos cuadrados parciales (Partial least squares, PLS por sus siglas en inglés) a través del software SmartPLS versión 2.0.M3 (Ringle et al., 2005), fue aplicada. La elección de PLS es justificada por la naturaleza exploratoria del estudio; esto debido a que una parte del instrumento de medición fue desarrollado parcialmente por las investigadoras, sin utilizar escalas validadas $o$ desarrolladas por otros autores.

\section{ANÁLISIS DE RESULtAdos}

\section{Perfil de los entrevistados}

Un poco más de la mitad de los clientes entrevistados fueron varones $(54.5 \%)$; la gran mayoría se encuentra en un rango de edad de 20 a 39 años $(93.0 \%)$. Además, casi la totalidad de estos saben leer y escribir $(98.0 \%)$, pertenecientes a clase baja (estrato D, correspondiente al penúltimo de cuatro niveles socioeconómicos propuestos por Sigmarket, 2008), con un ingreso promedio de $\$ 575$ dólares. Estos son atendidos por un banco a través de algún tipo de crédito y/o cuenta de cheques o ahorro. 


\section{ANálIsIS Con mínImos CUADRAdos parciales (PLS)}

Henseler et al. (2008) proponen validar el modelo en dos etapas: 1) revisión de las mediciones formativas y reflectivas a través de diversos procedimientos y 2) validación de la porción estructural.

\section{Modelos de MEDICIÓN}

El modelo de medición reflectivo es evaluado al verificar la fiabilidad del ítem individual, la consistencia interna o fiabilidad del constructo, el análisis de la varianza promedio extraída y la validez discriminante (Esposito et al., 2010). La significancia de los estimadores de los parámetros (pesos) para cada indicador formativo, evaluados como en una ecuación de regresión, es interpretada como un coeficiente de validez (Bollen, 1989). La significancia del peso fue evaluada con el procedimiento bootstrap; aun cuando los resultados de 500 submuestras evidencien que ciertos ítems no son significativos con un nivel de confianza del $90 \%$, estos son retenidos en el modelo para su valoración, dada la naturaleza exploratoria del estudio y según lo recomendado por Chin (1998). Cenfetelli y Bassellier (2009) sugieren considerar los constructos formativos basados en: 1) examinación de multicolinealidad, 2) el número de indicadores, 3) la posible ocurrencia de pesos negativos y positivos de los indicadores, 4) la contribución absoluta vs. la contribución relativa del indicador formativo, 5) los efectos de la red nomológica y 6) los posibles efectos de usar PLS contra las técnicas SEM basadas en covarianza.

La presencia de multicolinealidad puede representar un problema en indicadores reflectivos y formativos. Dado que los indicadores de los constructos del modelo conceptual (Figura 1), no se distribuyen normal (Prueba de Shapiro Wilks, Ho: la variable se distribuye normal), se calcula la rho de Spearman (Garson, 2009b). Únicamente la correlación entre los dos indicadores de IAS (ítem 26 y 27) es excesiva, mayor a 0.90 indicando problemas de colinealidad (Garson, 2010) (resultados no mostrados); tal que para no perder información al eliminar alguno de los ítems, los puntajes de ambos indicadores se suman, proponiendo una nueva variable resultado de dicha operación (ítem 28). Además, al ana- 
lizar los enunciados del modelo conceptual propuesto (Figura 1), se concluye que no existe traslape de ítems. Por lo cual, podría asumirse lo sugerido por Sekaran (2000), que cada variable es lo suficientemente distinta para medir diferentes variables en el mismo constructo.

\section{MODELOS DE MEDICIÓN REFLECTIVOS}

Otra forma de evaluar multicolinealidad es a través del FIV (factor de inflación de la varianza, VIF por sus siglas en inglés). El punto de corte en indicadores reflectivos corresponde a un FIV mayor a 10 (Henseler et al., 2008). El paquete estadístico SPSS versión 15.0, fue utilizado para calcular el FIV, a través de regresiones múltiples para cada grupo de indicadores pertenecientes a una medición reflectiva (variable independiente); la variable dependiente fue elegida arbitrariamente como un indicador de alguna variable endógena (Seidel y Back, 2009). Todos los constructos reflectivos endógenos, que poseen más de un indicador en el modelo teórico, presentan variables con FIV menores a 10; indicando que no existen problemas de colinealidad (resultados no mostrados).

El tamaño de la carga es un criterio utilizado para evaluar la fiabilidad del indicador, con el constructo que éste pretende medir (Seidel y Back, 2009). A base de la regla propuesta por Hulland (1999) de conservar los ítems con cargas de 0.7 o más, todos los indicadores de los modelos de medición reflectivos, con más de un indicador, alcanzan el nivel aceptable de fiabilidad (Figura 2).

La validez de convergencia es evaluada con el valor del Promedio de Varianza Extraída (Average Variance Extracted, AVE por sus siglas en inglés), el cual debe ser mayor a 0.5 (Criterio de Fornell-Larcker) (Seidel y Back, 2009). En el modelo, el constructo reflectivo con más de un indicador SPCB, presenta un valor por arriba del límite (0.6915) (resultados no mostrados). La validez de convergencia también es demostrada cuando los ítems cargan de manera alta en sus factores asociados (carga mayor a 0.5 ) y estas deben ser mayores en el constructo asignado que en cualquier otro; todos ellos satisfacen dicho criterio, aun cuando el ítem 19 y 21 de SPCB exhiben cargas muy altas en constructos formativos (Seidel y Back, 2009) (Tabla 2). 


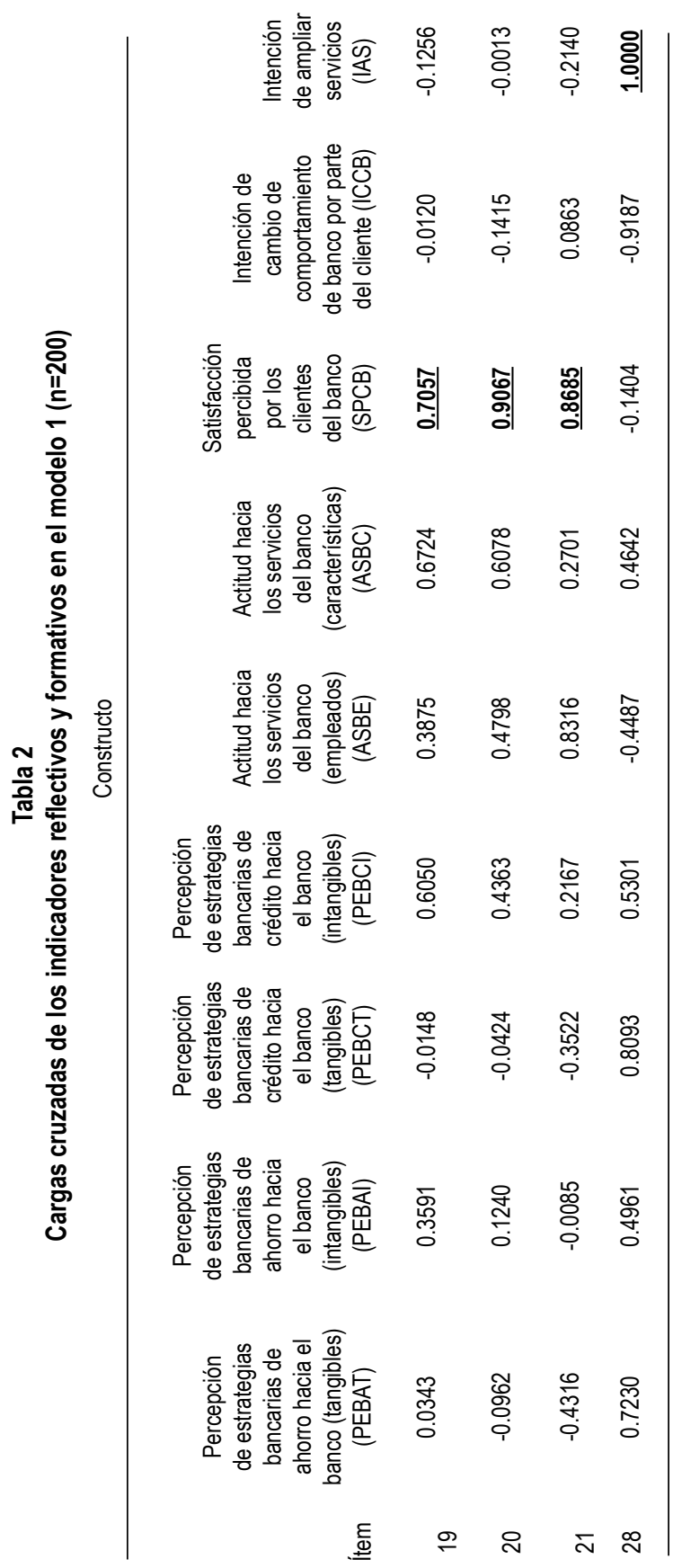


El coeficiente Rho de Dillon-Goldsteins (índice de fiabilidad compuesta) es utilizado para evaluar la consistencia interna (Fornell y Larcker, 1981). En el modelo final propuesto (Figura 2 2), el índice de fiabilidad compuesta para SPCB (constructo reflectivo con dos o más variables), es 0.8963 (resultados no mostrados), excediendo el valor mínimo aceptable de 0.70 (Seidel y Back, 2009; Hairt et al., 1998).

Fornell y Larcker (1981) sugieren que un puntaje de 0.5 del AVE (por sus siglas en inglés), indica un nivel aceptable de validez discriminante. El AVE (por sus siglas en inglés) para el constructo reflectivo SPCB es 0.6915 , por arriba del valor aceptable. Tal validez, es demostrada también cuando la raíz cuadrada de AVE de cada constructo es mayor que la correlación con cualquier otra variable latente (Seidel y Back, 2009; Deli y Lenz, 2008); tal criterio se cumple, sugiriendo validez discriminante (Seidel y Back, 2009; Duarte y Raposo, 2010) y convergente aceptable (Duarte y Raposo, 2010) (resultados no mostrados).

Chin (1998), Seidel y Back (2009), afirman que la validez discriminante se cumple al comparar las cargas cruzadas de los indicadores asignados al constructo reflectivo, contra el resto de ellas. En el modelo, no se revelan problemas de validez discriminante, dado que todos los indicadores muestran cargas mayores en su respectivo constructo con respecto a otro constructos reflectivos (Duarte y Raposo, 2010) (Tabla 2). Sin embargo, en la variable latente SPCB, el ítem 19 también carga fuertemente en ASBC; el ítem 21 presenta carga alta en el constructo ASBE (Tabla 2). En IAS, el ítem 28 presenta una carga excesiva en ICCB; siendo la diferencia menor al $10 \%$ (Tabla 2) (Seidel y Back, 2009).

\section{MODELOS DE MEDICIÓN FORMATIVOS}

\section{EXAMINACIÓN DEL NÚMERO DE INDICADORES}

Cenfetelli y Bassellier (2009) mencionan que el número de indicadores posee implicaciones importantes en la significancia estadística y por lo tanto en la magnitud de sus pesos. Estos sugieren agrupar los ítems en 2 o más constructos con efectos separados, incrementando la probabilidad de cualquier indicador de ser significativo. Los constructos PEBA, PEBC y ASB, presentan 6 
indicadores, por lo que estos se dividen en dos variables latentes; generando nuevas relaciones hipotéticas (Figura 2).

\section{EXAMINACIÓN DE MULTICOLINEALIDAD}

Una forma de analizar qué tan confiables son los estimadores de los paths (o caminos) para los constructos formativos, es el análisis de multicolinealidad a través del VIF (por sus siglas en inglés) (Seidel y Back, 2009). Los resultados indicaron VIF mayores a 1.5 (punto de corte) para todos los constructos formativos del modelo, con excepción de las variables latentes: PEBAI, PEBCT y ASBE (resultados no mostrados). El índice de condición y las proporciones de varianza, confirman tales complicaciones en todos los constructos formativos (punto de corte: mayor a 15 y mayor o igual a 0.5 , respectivamente) (Garson, 2010). Para prevenir problemas de multicolinealidad en los constructos formativos del modelo, se aplicó un análisis de componentes principales con rotación varimax para generar componentes que no se correlacionen (Malhotra, 2004). Los puntajes generados fueron utilizados como nuevas variables de los constructos correspondientes.

\section{EXAMINACIÓN DE OCURRENCIA DE PESOS DE INDICADORES NEGATIVOS Y POSITIVOS}

En el modelo propuesto los indicadores formativos con pesos negativos (ítem 1, 5, 10, 12 y 13), coinciden en signos con sus cargas (resultados no mostrados). Tal presencia de signos positivos y negativos, simplemente sugiere revertir la escala de dichos ítems con pesos negativos, para facilitar la interpretación (Nunnally y Bernstein, 1994); no obstante, en esta investigación se decidió respetar la dirección positiva de las escalas Likert examinadas. Dado que no existe repetición de ítems, se conservarán los indicadores de todos los constructos formativos del modelo propuesto, aún aquellos que poseen peso negativo no significativo (Fig. 2); una relación sustancial debe ser verificada (Seidel y Back, 2009).

\section{COMPARACIÓN DE LA CONTRIBUCIÓN ABSOLUTA VS. LA CONTRIBUCIÓN RELATIVA DEL INDICADOR FORMATIVO}

El análisis de la contribución absoluta del indicador (carga) contra la contribución relativa de éste (peso), mostró que en el modelo 
propuesto, los ítems $1,4,5,6,7,10,12,13,14,15$ y 25 , no presentan una contribución relativa significativa aún con un nivel del 15\% (Figura 2); siendo la contribución absoluta no significativa aún con un alpha del 15\%, para todos ellos (resultados no mostrados). Lo anterior sugiere que la relevancia teórica de los ítems mencionados, debe ser cuestionada (Cenfetelli y Bassellier, 2009). Sin embargo, el desarrollo de las escalas de medición utilizadas en esta investigación, se basó en la literatura; debido a que dichos ítems contribuyen a la validez de contenido de los constructos, estos son retenidos (Bollen y Lennox, 1991; Petter et al., 2007) y se propone analizarlos a profundidad con investigación futura.

\section{EVALUACIÓN DE LOS EFECTOS DE LA RED NOMOLÓGICA}

La validez en un constructo formativo es apoyada con el índice formativo relacionado con la estructura nomológica (Diamantopoulos y Winklhofer, 2001). La validación nomológica es examinada únicamente para los constructos formativos, ASBE, ASBC e ICCB; esto es debido a la necesidad de una relación teórica con un constructo reflectivo. Los resultados muestran que dichos constructos están relacionados significativamente con un alpha de 0.05 (valor $\mathrm{t}<1.96$ ), con alguno de los constructos reflectivos (SPCB o IAS) (Figura 2).

\section{Posibles efectos de usar PLS contra las técnicas SeM basadas en COVARIANZA}

El valor de los pesos podría estar incrementado al haber utilizado la técnica PLS (Cenfetelli y Bassellier, 2009). No obstante, la elección de la técnica de modelos de ecuaciones estructurales basados en varianza (PLS) se justifica por el objetivo de la investigación.

\section{Modelo estructural}

Dado que el principal objetivo de PLS es la predicción, la bondad de ajuste del modelo es establecida a base de la magnitud de los coeficientes paths estructurales y la predictibilidad combinada $\left(\mathrm{R}^{2}\right)$ de sus constructos exógenos (Chin, 1998). Falk y Miller (1992) sugieren que $\mathrm{R}^{2}$, debería ser mayor a 0.1 . En el modelo propuesto (Figura 2) el constructo dependiente final, la intención de ampliar los servicios (IAS), presenta un $\mathrm{R}^{2}$ de 0.8703 . Es decir, el $87.03 \%$ 
de su varianza es explicada sustancialmente (Chin, 1998) por SPCB, ICCB, ASBE y ASBC, así como por PEBAT, PEBAI, PEBCT y PEBCI. Sólo un constructo no cumple con el criterio de Falk y Miller (1992). Únicamente 8 coeficientes paths principales de un total de 13, son mayores a 0.2 (estándar mínimo de significancia según Chin (1998)); es decir el $61.40 \%$. Sólo 6 coeficientes (46.15\%) fueron significativos con al menos un nivel de confianza del 95\%. Sin embargo en la hipótesis 1 (H1a), el signo resultante es contrario al propuesto (Figura 2).

Figura 2

Modelo final de ampliación a la bancarización, con cargas para constructos reflectivos, pesos para constructos formativos y coeficientes path

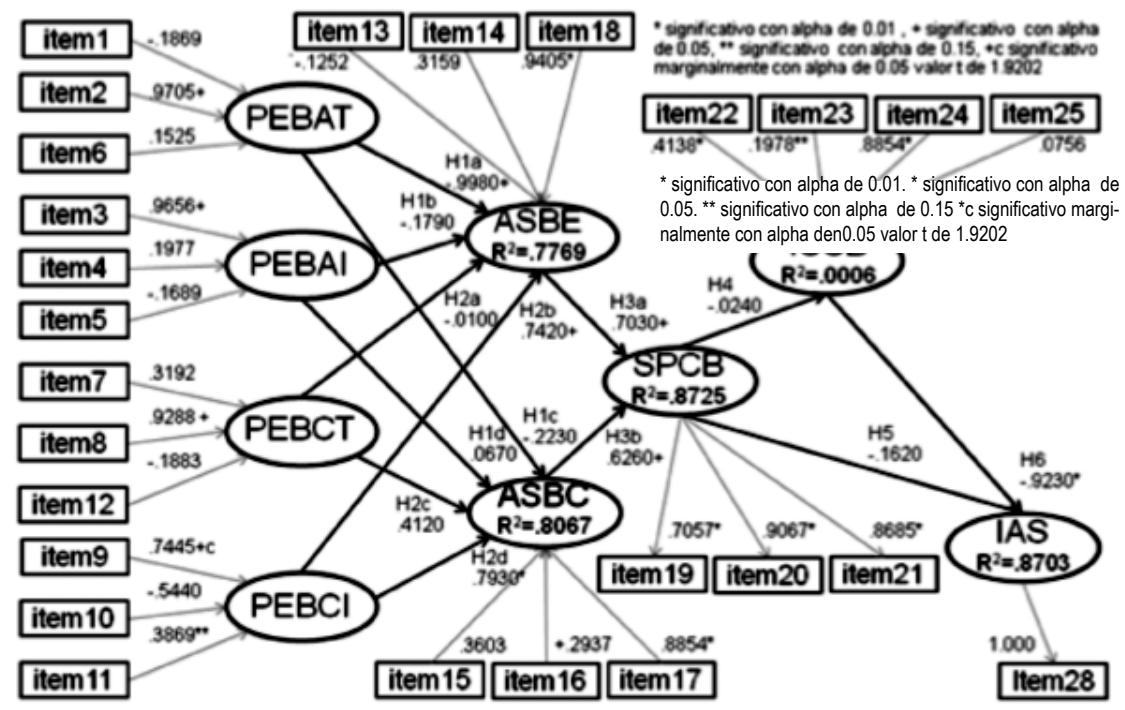

PEBAT $=$ Percepción de estrategias bancarias de ahorro hacia el banco (tangibles). PEBAI = Percepción de estrategias bancarias de ahorro hacia el banco (intangibles). PEBCT = Percepción de estrategias bancarias de crédito hacia el banco (tangibles) PEBCI = Percepción de estrategias bancarias de crédito hacia el banco (intangibles) ASBE = Actitud hacia los servicios de banco (empleados). $A S B C=$ Actitud hacia los servicios de banco (características ) SPCB = Satisfacción percibida por los clientes del banco. ICCB =Intención del comportamiento de cambio de banco por parte del cliente. IAS = Intención para ampliar servicios.

En general, con estos resultados se concluye que, el modelo con 9 constructos, para personas bancarizadas (Figura 2), predice la intención de ampliar servicios (IAS) con las variables latentes: 1) PEBAT, 2) PEBCI, 3) ASBE, 4) ASBC y 5) ICCB. El comportamiento 
de cambio de banco por parte del cliente (ICCB) es el factor más importante en términos de influencia directa negativa, en la intención de ampliar servicios (IAS) (coeficiente path $=-0.9230$, Fig. 2).

Asimismo, el posible efecto mediador de $I C C B$ en la relación de $S P C B$ con $I A S$, es examinado a través de la aproximación de Iacobucci y Duhachek (2003) donde $\mathrm{H}_{\mathrm{o}}$ : No existe efecto indirecto de $S P C B$ en $I A S$ vía el constructo $I C C B$. Se concluye que no existe suficiente evidencia de tal efecto indirecto (estadístico Z (Sobel, 1982) con valor de 0.1337 no es mayor a 1.96).

\section{Discusiones y Conclusiones}

La investigación reveló factores y su interacción a través de un modelo relacionado al proceso de intención de ampliar servicios bancarios en consumidores de bajos ingresos en Monterrey, México.

La intención de cambio de comportamiento por parte del cliente es un factor que afecta de forma crucial la intención de ampliar los servicios bancarios con la institución financiera. La fuerza de la intención radica en que es un predecesor de la conducta. Los costos en los servicios y créditos así como los efectos de la tasa de interés son determinantes en la posible pérdida de relaciones de largo plazo entre cliente y prestador de servicio. La gerencia de la institución financiera debe buscar la forma de incrementar las barreras a la generación de la intención de cambio de comportamiento, a fin de hacer menos atractivo el cambio. Algunos autores (Berry y Parasuraman, 1991) sugieren que debe acrecentarse la dependencia en la relación de largo plazo y, aunque la estrategia más común para ello se realiza vía la ampliación del portafolio de inversiones en los clientes de altos ingresos, también puede experimentarse con la oferta de servicios financieros básicos a clientes con ingreso bajo.

A pesar de que la satisfacción es un predictor de las intenciones de cambio de comportamiento y ampliación de servicios (Moutinho y Meidan, 1989; Manrai y Manrai, 1993), en esta investigación empírica ambas resultaron no significativas. Esto no demerita la importancia de la satisfacción del cliente ya que las percepciones y las actitudes desarrolladas afectan la satisfacción. Los resultados refuerzan la relación probada en otros trabajos desarrollados en la industria 
bancaria entre la actitud y la satisfacción del cliente (Moutinho y Smith, 2000). El efecto indirecto vía intención del comportamiento de cambio de banco (ICCB) no fue evidenciado, difiriendo de lo establecido en otros estudios (Lees, Garland y Wright, 2007). Es posible que otros aspectos como los incentivos a la informalidad, la falta de cultura crediticia, así como los bajos niveles de educación actúen como barreras en las familias de bajos ingresos para acceder a más servicios de las instituciones financieras (Carbajal, 2008).

Otros factores que contribuyen a explicar el proceso de intención de ampliar los servicios con su banco y que presentan contribuciones indirectas pero significativas y robustas son: 1) la percepción de las estrategias bancarias de ahorro (tangibles) hacia el banco, 2) percepción de las estrategias bancarias de crédito (intangibles) hacia el banco, 3) actitud hacia los servicios del banco enfocados en sus empleados y, 4) actitud hacia los servicios del banco respecto a sus características. La actitud que los clientes tienen hacia los servicios vinculados al contacto humano con los empleados bancarios se ve afectada inversamente por las percepciones que se tienen sobre las facilidades tangibles como ubicación, uso de tecnología en cajeros y banca electrónica, así como productos para facilitar el ahorro. Estos aspectos requieren un nulo o casi nulo contacto con ejecutivos y cajeros del banco, por lo que es posible que cuando se incremente el uso y se tenga una percepción positiva de estos tangibles, entonces se reduzca la predisposición favorable hacia los encuentros humanos de servicio en la sucursal.

Por otro lado, la actitud hacia los empleados se ve influida por la percepción de aspectos intangibles que facilitan el acceso al crédito (como la tasa de interés, el horario de atención o los tipos de crédito que puede ofrecer el ejecutivo al cliente). Estos aspectos intangibles vinculados al crédito a su vez inciden sobre la actitud hacia los servicios del banco, es decir características propias del banco como número de sucursales, ubicación y seguridad. Esto coincide con los aspectos que sustentan el valor agregado como el servicio, las sucursales, la marca y el talento del recurso humano (Lai, 1995; Flint et al., 2002). Los esfuerzos orientados a lograr la calidad suelen ser recompensados en diferenciación competitiva. A pesar de que las tendencias apuntan a fortalecer una banca electrónica orientada a 
los clientes de clase media y alta en el país, las iniciativas orientadas a los clientes de clase baja deben facilitar el acceso físico a los servicios financieros y, también es necesario incrementar la disposición y facilitación de la información a un mercado que enfrenta más barreras relacionadas con el conocimiento y uso de los servicios financieros.

Las entidades bancarias orientadas a la clase baja deben afinar sus prácticas financieras y comerciales relacionadas con los productos y servicios ofrecidos, adaptar las herramientas tecnológicas, los formatos, la papelería y los contenidos. El enfoque debe estar orientado hacia la claridad y la comprensión de beneficios, los costos y riesgos de la contratación de servicios por parte de los clientes con baja educación financiera, a fin de reducir la incertidumbre que podría llevar a la intención de cambio de banco, incrementando la intención de ampliar los servicios bancarios con la institución.

Los resultados de este estudio presentan ciertas limitaciones. Primero, esta investigación fue realizada solamente en la ciudad de Monterrey, capital del estado de Nuevo León en el norte de México, por lo que los resultados podrían diferir al aplicarse en otras regiones del país. Segundo, el modelo debe ser examinado con escalas más refinadas para algunos constructos con el fin de solucionar problemas metodológicos asociados con la significancia estadística de dichas mediciones (Duarte y Raposo, 2010). Tercero, al ser una muestra no probabilística, los resultados de las pruebas de hipótesis deben tomarse con cautela. En estudios posteriores, es recomendable incluir otros constructos relevantes, tales como la lealtad del cliente al banco, el impacto del tiempo en la calidad de la relación clientebanco y la recuperación del servicio. Es aconsejable que este estudio sea replicado con otras muestras de consumidores bancarizados del segmento de bajos ingresos considerando otros contextos en México o en América Latina e introduciendo otras variables relevantes para la mercadotecnia desarrollada por las instituciones bancarias. 


\section{Referencias}

Bearden, W., Netemeyer, R. y Teel, J. (1989). Measurement of consumer susceptibility to interpersonal influence. Journal of Consumer Research, 15, 473-481.

Bejou, D. y Palmer, A. (1998). Service failure and loyalty: an exploratory empirical study of airline customers. Journal of Services Marketing, 12(1), 7-22.

Berry, L. L. y Parasuraman, A. (1991). Marketing service: Competing through quality. New York: The Free Press.

Bitner, M. J. y Hubbert, A. R. (1994). Encounter satisfaction versus overall satisfaction versus quality: The customer's voice. En R. T. Rust, y R. L. Oliver (Eds.), Service quality: New directions in theory and practice (pp.72-94). Thousand Oaks, CA: Sage.

Bollen, K. A. (1989). Structural equations with latent variables (1st ed.). Malden, MA: Wiley-Interscience.

Bollen, K. A. y Lennox, R. (1991). Conventional wisdom on measurement: A structural equation perspective. Psychological Bulletin, 110(2), 305-314.

Carbajal, J. (2008). Educación financiera y bancarización en México. México: CEEDE.

Cenfetelli T. R. y Bassellier G. (2009). Interpretation of formative measurement in information systems research. MIS Quarterly, 33(4), 689-707.

Chin, W. W. (1998). The partial least squares approach to structural equation modeling. En G. Marcoulides (Ed.), Modern Methods for Business Research (pp. 295-336). Mahwah, NJ: Lawrence Erlbaum.

Clemes, M. D., Gan, C. y Zheng, L. Y. (2007). Customer switching behavior in the New Zealand banking industry. Banks and Banks System, 2(4), 50-66.

Crosby, L., Evans, K. y Cowies, D. (1990). Relationship quality in services selling: An interpersonal influence perspective. Journal of Marketing, 54(3), 68-81. 
Dabholkar, P. A. (2000). Technology in service delivery: Implications for self-service and service support. En T. A. Swartz y D. Iacobucci (Eds.), Handbook of Services Marketing and Management (pp. 103110). Thousand Oaks: Sage Publications.

Diamantopoulus, A. y Winklhofer, H. (2001). Index construction with formative indicators: An alternative to scale development. Journal of Marketing Research, 2(38), 269-277.

Deli , D. y Lenz H-J. (2008). Benchmarking user perceived impact for web portal success evaluation. JIOS, 32(1), 1-14.

Duarte, P. A. y Raposo, M. L. B. (2010). A PLS model to study brand preference: An application to the mobile phone market. En V. Esposito Vinzi, W. W. Chin, J. Henseler y H. Wang (Eds.), Handbook of partial least squares: Concepts, methods and applications. Heidelberg, Germany: Springer.

Esposito Vinzi V., Chin W. W., Henseler J. y Wang H. (Eds.). (2010). Handbook of partial least squares: Concepts, methods and applications. Heidelberg, Germany: Springer.

Falk, R. F. y Miller, N. B. (1992). A primer for soft modeling. Ohio: The University of Akron Press.

Fassott, G. (2006). Operationalisierung latenter Variablen in Strukturgleichungsmodellen: Eine Standortbestimmung. Zfbf, 58, Februar, 67-88.

Flint, D. J., Woodruff, R. B. y Gardial, S. F. (2002). Exploring the phenomenon of customer's desired value change in a businessto-business context. Journal of Marketing, 66, 102-117.

Fornell, C. y Larcker, D. (1981). Evaluating structural equation models with unobservable variables and measurement error. Journal of Marketing Research, 18, 39-50.

Galán, V. (2007). Banco Wal-Mart abre cuentas de 50 pesos. Recuperado de http://www.cnnexpansion.com/negocios/2007/11/13/ wal-mart-tendra-cuentas-de-50-pesos

Gammacurta, G. (2006). Cómo venderles a las mujeres. Fortuna. Recuperado de http://www.fortuna.uol.com.ar/edicion_0138/ nota_tapa/nota_tapa.htm

Garland, R. (2002). Estimating customer defection in personal retail banking. The International Journal of Bank Marketing, 20(7), 317325 . 
Garrido, C. y García, G. (2009). Bancarización centrada en el crédito al consumo y opacidad regulatoria. Una nota sobre el sistema financiero mexicano ante la crisis global. El Cotidiano, 24(157), 37-48.

Garrido, C., García, G. y Morales, R. (2011). Los esquemas de corresponsalía bancaria en México: ¿solución al problema de acceso a servicios financieros? Análisis Económico, XXVI (61), 117-137.

Garson, G. D. (2009a). Data levels and measurement. Statnotes: Topics in multivariate analysis. Recuperado de http://faculty.chass. ncsu.edu/garson/PA765/datalevl.htm

Garson, G. D. (2009b). Correlation. Statnotes: Topics in multivariate analysis. Recuperado de http:/ / faculty.chass.ncsu.edu/garson/ PA765/correl.htm\#assume

Garson, G. D. (2010). Multiple regression. Statnotes: Topics in multivariate analysis. Recuperado de http://faculty.chass.ncsu. edu/garson/PA765/regress.htm\#multicollinearity

Hair, J. F., Jr., Anderson, R. E., Tatham, R. L. y Black, W. C. (1998). Multivariate data analysis ( $5^{\text {th }}$ ed.). London: Prentice Hall International.

Hellier, P., Geursen, G., Carr, R. y Richard, J. (2003). Customer repurchase intention. A general structural equation model. European Journal of Marketing, 37(11/12), 1762-1800.

Henseler, J., Ringle, C. M. y Sinkovics, R. R. (2008). The use of partial least squares path modeling in international marketing. Advances in International Marketing, 19, 1-43.

Hocutt, M. (1998). Relationship dissolution model: antecedents of relationship commitment and the likelihood of dissolving a relationship. International Journal of Service Industry Management, 9(2), 189-200.

Hoffman, K. D. y Bateson, J. (2006). Fundamentos de marketing de servicios. México: Thomson.

Hulland, J. (1999). Use of partial least squares (PLS) in strategic management research: a review of four recent studies. Strategic Management Journal, 20, 195-204.

Iacobucci, D. y Duhachek, A. (2003). Mediation analysis (round table). Presentación en ACR Conference, Toronto. 
Jarvis, C. B., MacKenzie, S. B. y Podsakoff, P. M. (2003). A critical review of construct indicators and measurement model misspecifications in marketing and consumer research. Journal of Consumer Research, 30, 199-218.

Johnson, M. D. y Gustafsson, A. (2000). Improving customer satisfaction, loyalty and profit: An integrated measurement and management system. San Francisco, CA: Jossey-Bass.

Keaveney, S. (1995). Customer switching behavior in service industries: An exploratory study. Journal of Marketing, 59(2), 71-82.

Kim, W. y Mauborgne, R. (2005). La estrategia del océano azul. Bogotá: Grupo Editorial Norma.

Kline, R. B. (2010). Principles and practice of structural equation modeling ( $3^{\text {rd }}$ ed.). New York: Guilford Press.

Lai, A. W. (1995). Consumer value, product benefits and customer value: A consumption behavior approach. Advances in Consumer Research, 22, 381-388.

Lam, R. y Burton, S. (2006). SME banking loyalty: A qualitative study in Hong Kong. International Journal of Bank Marketing, 24(1), 37-52.

Lam, R., Burton, S. y Lo, H-P. (2009). Customer tradeoffs between key determinants of SME banking loyalty. International Journal of Bank Marketing, 27(6), 428-445.

Lees, G., Garland, R. y Wright, M. (2007). Switching banks: Old back gone but not forgotten. Journal of Financial Services Marketing, 12(2), 146-156.

Lovelock, C. (1997). Mercadotecnia de servicios. México: Prentice-Hall.

Malhotra, N. (2004). Investigación de mercados. Un enfoque aplicado. México: Prentice Hall.

Manrai, L. A. y Manrai, A. K. (1993). Marketing in Romania: the challenges of the transition from a centrally-planned economy to a consumer-oriented economy. European Journal of Marketing, 27(11/12), 102-120.

Mercado, S. (2007). Mercadotecnia de servicios. México: PAC.

Moutinho, L. (1992). Customer satisfaction measurement: prolonged satisfaction with ATMs. International Journal of Bank Marketing, 10(7), 30-37. 
Moutinho, L. y Meidan, A. (1989). Bank customers' perceptions innovation and new technology. International Journal of Bank Marketing, 7(2), 22-27.

Moutinho, L.ySmith, A. (2000). Modelling bank customer satisfaction through mediation of attitudes towards human and automated banking. International Journal of Bank Marketing, 18(3), 124-134.

Murdock G. y Franz, L. (1983). Habit and perceived risk as factors in the resistance to the use of ATMs. Journal of Retail Banking, 5(2), 20-29.

Nunnally, J. C. y Bernstein, I. (1994). Psychometric Theory (3 ${ }^{\text {ra }}$ ed.). New York: McGraw-Hill.

Oliver, R. L. (1997). Satisfaction: A behavioral perspective on the customer. Boston: McGraw-Hill.

Petter, S., Straub, D. y Rai, A. (2007). Specifying formative constructs in information systems research. MSI Quarterly, 31(4), 623-656.

Ringle, C. M., Wende, S. y Will, A. (2005). SmartPLS 2.0 (Beta). University of Hamburg. Recuperado de http:/ /www.smartpls.de

Rojas, L. (2006). El acceso a los servicios bancarios en América Latina: Identificación de obstáculos y recomendaciones. Center for Global Development. Recuperado de http:/www.cgdev.org/ doc/experts/Serv_Bancarios_AL.pdf

Schiffman, L. G. y Kanuk, L. L. (2001). Comportamiento del consumidor ( $7^{\mathrm{a}}$ ed.). México: Prentice Hall.

Seidel, G. y Back, A. (2009). Success factor validation for global ERP programmes. Trabajo presentado en la $17^{\text {th }}$ European Conference on Information Systems. Manuscript ID: ECIS2009-0098.R1.

Sekaran, U. (2000). Research methods for business: A skill-building approach ( $3^{\text {ra }}$ ed.). New York: Wiley.

Sigmarket. (2008). Mercados potenciales, 2008. El mercado de los consumidores mexicanos por niveles socioeconómicos. México: Sigmarket.

Smith, A. M. (2000). The impact of scale characteristics on the dimensionality of the service quality construct. The Service Industries Journal, 20(3), 167-190. 
Sobel, M. E. (1982). Asymptotic confidence intervals for indirect effects in structural equation models. En S. Leinhardt (Ed.), Sociological Methodology 1982 (pp. 290-312). Washington DC: American Sociological Association.

Valencia, T. (2005). Ofrecer un servicio de calidad. Recuperado de http://www.mailxmail.com/curso-ofrecer-servicio-calidad/ que-es-actitud-servicio

Walsh, S., Gilmore, A. y Carson, D. (2004). Managing and implementing simultaneous transaction and relationship marketing. The International Journal of Bank Marketing, 22(7), 468-483.

Zamarripa, G. (2008). Regulación y competencia en el sector bancario: Nuevos intermediarios, bancos tienda y banca de nicho. Seminario de Modernización e Inclusión Financiera en América Latina. México: Banxico-Cemla. 


\section{APÉndice}

\section{Tabla 1}

\section{Medición de los seis constructos del modelo conceptual}

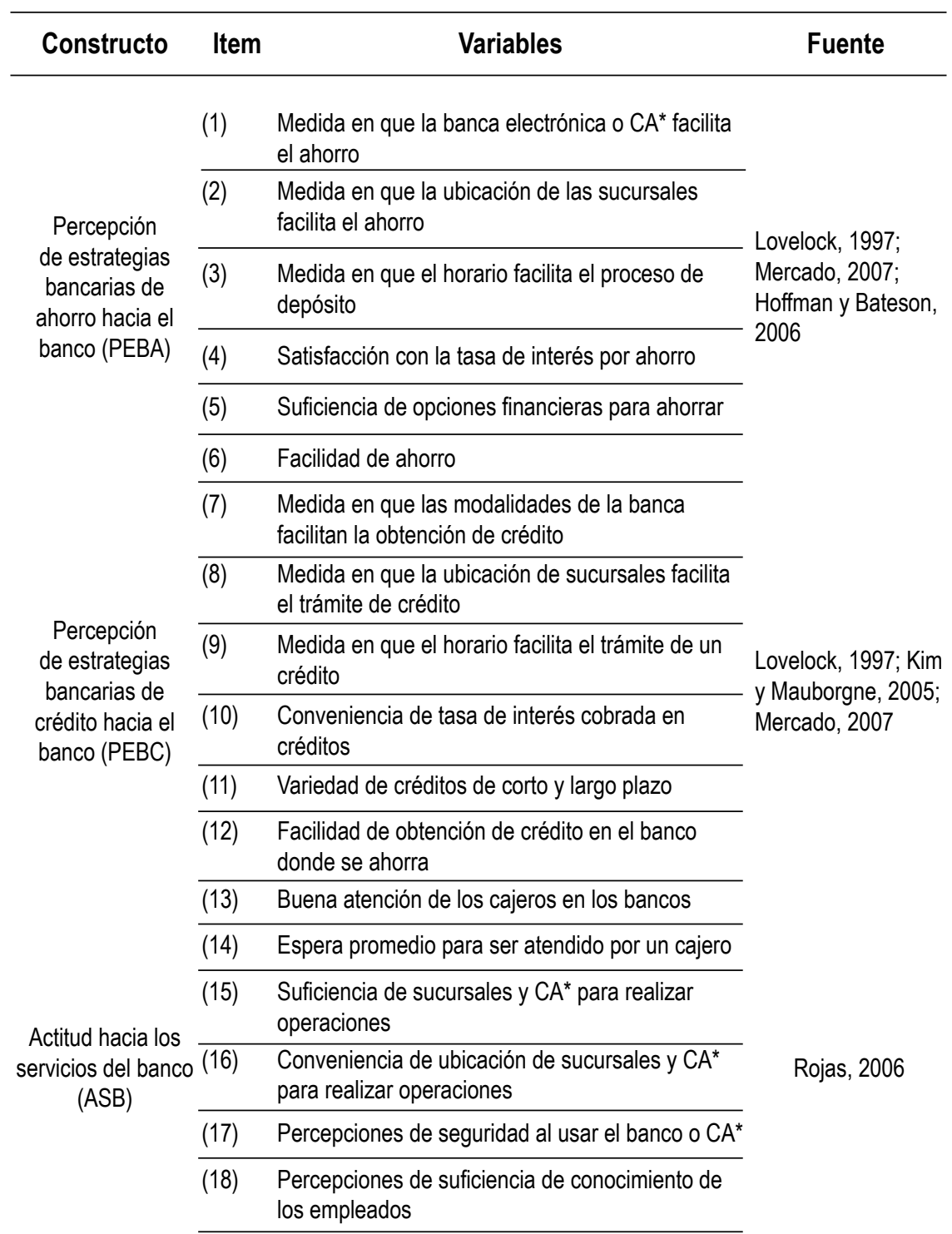


PROPUESTA DE UN MOdelo ORIENTADO A LA INTENCIÓN DE AMPLIAR SERVICIOS DE AHORRO Y CRÉDITO...

Cont. Tabla 1

(19) Satisfacción de servicios recibidos por cajeros humanos

Satisfacción percibida por los clientes del banco (SPCB)

(20) Satisfacción de servicios recibidos a través de Johnson y banca automática Gutafsson, 2000

(21) Satisfacción general con el banco actual

(22) Efectos de la tasa de interés en la cuenta de ahorro, en la ICCB

Intención de

comportamiento de cambio de banco (ICCB)

(23) Efectos de la tasa de comisiones o tasas de interés en créditos, en la ICCB
(24) Efecto de comisiones por retiro de ahorros, en la ICCB
(25) Efecto de comisiones por prepago de créditos, en la ICCB

Moutinho y Smith, 2000

(26) Percepción de diversidad de oferta en opciones

Intención de ampliar servicios

(IAS) financieras

(27) Percepción de cobertura de necesidades de ahorro y/o crédito

Carson, 2004

${ }^{*} \mathrm{CA}=$ cajeros automáticos 